\title{
A New Synthetic Spiroketal: Studies on Antitumor Activity on Murine Melanoma Model In Vivo and Mechanism of Action In Vitro
}

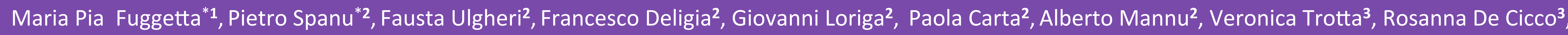
Adriano Barra ${ }^{3}$, Enrica Zona ${ }^{3}$, Franco Morelli ${ }^{* 3}$

${ }^{1}$ Istituto di Farmacologia Traslazionale - Consiglio Nazionale delle Ricerche, Roma, Italy

${ }^{2}$ Istituto di Chimica Biomolecolare - Consiglio Nazionale delle Ricerche, Sassari, Italy

${ }^{3}$ Istituto di Genetica e Biofisica A. Buzzati Traverso - Consiglio Nazionale delle Ricerche, Napoli, Italy

\section{- INTRODUCTION:}

Inspired by bioactive natural spiroketals, spirocyclic rigidified structures have demonstrated to be privileged scaffolds for the design of new tool compounds endowed with diversified biological activities. We synthesised the natural-like spiroketal, 2-hydroxy-8-methyl-1,7dioxaspiro[5.5] undec-3-en-5-one (5) that showed a potent anticancer activity against human cancer cells of different nature and histotype. ${ }^{1}$ In order to confirm the therapeutic potential of this molecule we verified in vitro and in vivo, in a syngenic murine melanoma model, that our product is able to induce cancer regression and growth inhibition.

\section{- SYNTHESIS OF THE SPIROKETAL 5:}

The synthesis of the spiroketalic stereoisomeric mixture $\mathbf{5}_{a, b}$ was carried out by using a two steps procedure based on 2-furyl ketone oxidation-rearrangement method. Because, in our previous experiments, the inhibition activity of the enantiomers was comparable, the steroisomeric mixture $\mathbf{5}_{\mathrm{a}, \mathrm{b}}$ was used as such for in vitro and in vivo experiments.

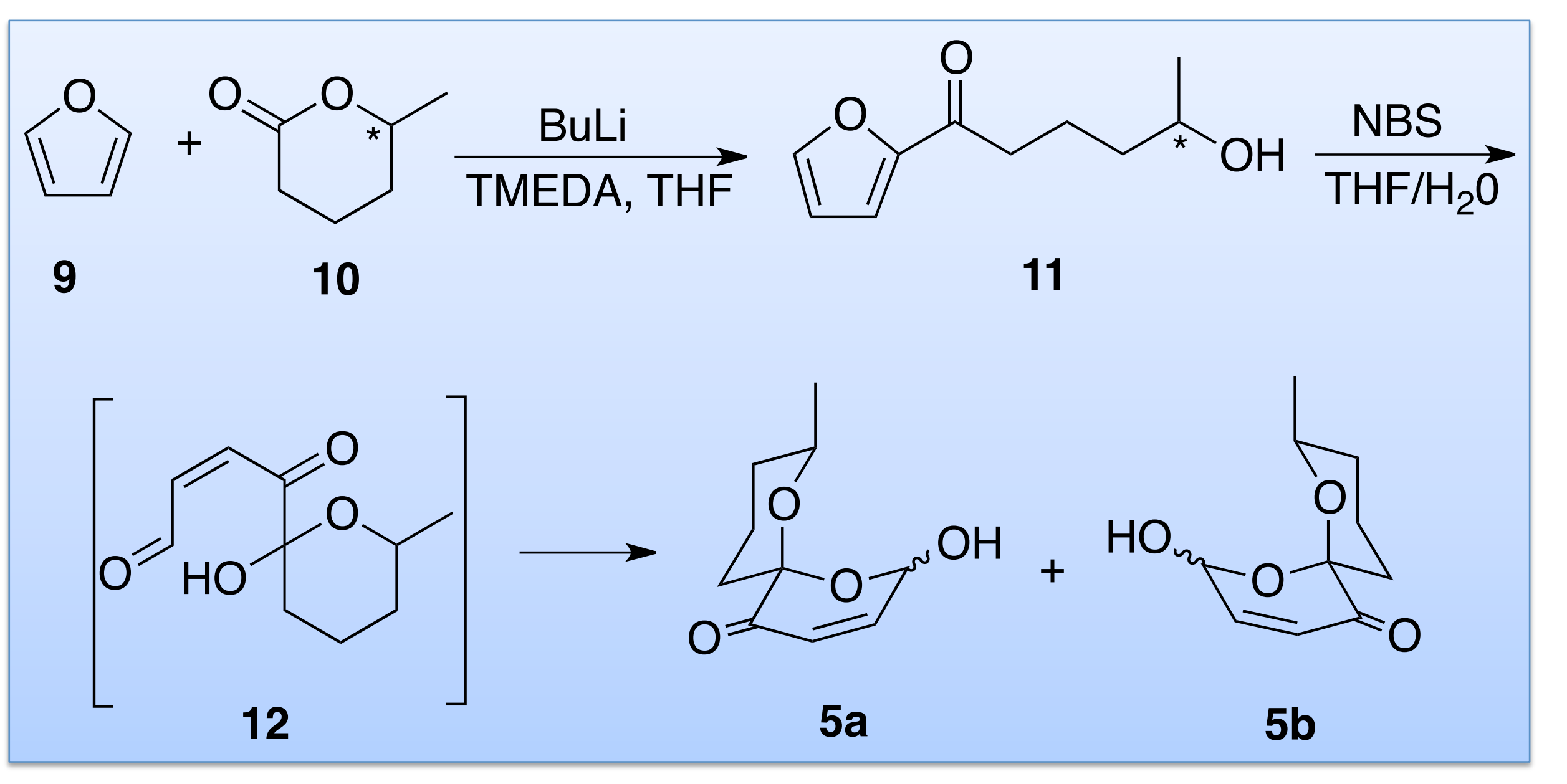

\section{- IN VITRO ACTIVITY:}

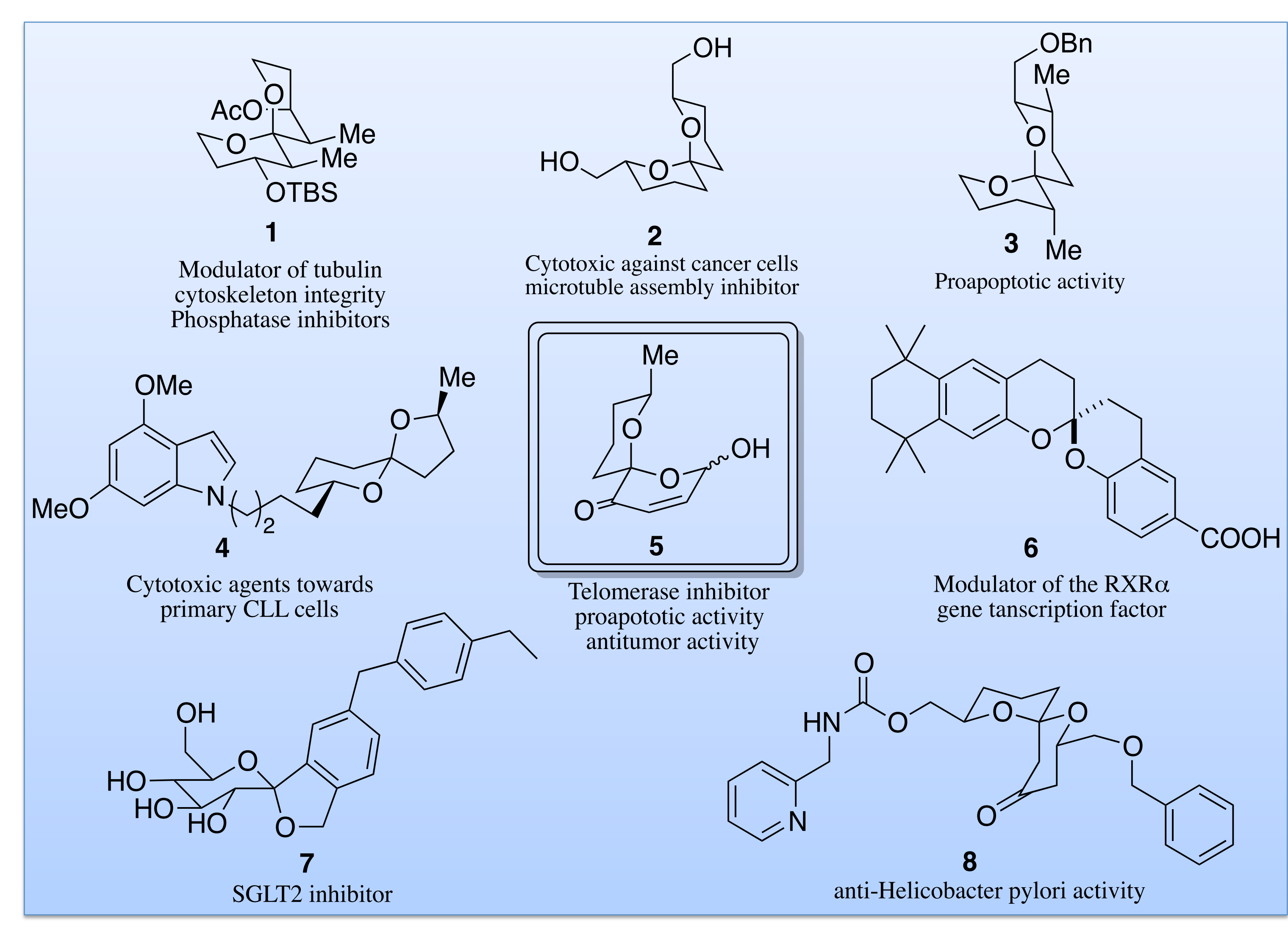

In the first set of experiments, the effect of spiroketal $\mathbf{5}_{a b}$ was evaluated in vitro on B16 cell growth in order to confirm on this murine model, the previously reported efficacy on human cell lines. Moreover, to acquire additional information on the origin of the antiproliferative activity, we have also studied the role of cell cycle modification, the apoptosis induction, the migration characteristics of B16 cultured cells, the gene expression of the hypoxia inducible factor $1 \alpha$ (HIF1 $\alpha$ ) in B16 murine melanoma cells and the modification of the actin structure and cytoskeleton conformation induced by $\mathbf{5}_{a, b}$
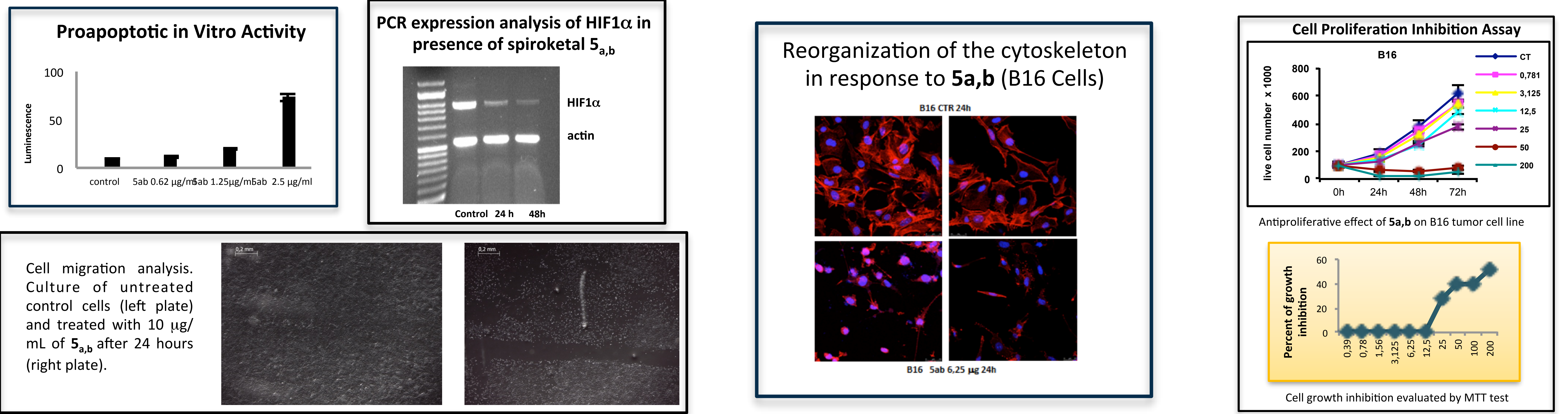

\section{- IN VIVO ACTIVITY:}

The assessment of the in vivo activity of the spiroketal $\mathbf{5}_{\mathrm{a}, \mathrm{b}}$ in a well-known B16/C57BL/6J syngenic model of murine melanoma was performed. The effect of this compound on cancer growth reported in the Figures, showed a tumor volume reduction with respect to the control.

\section{- CONCLUSION:}

We have identified the spiroketal $\mathbf{5}_{\mathbf{a}, \mathbf{b}}$ as a new promising anticancer agent with in vivo activity on a murine melanoma model. Compound $\mathbf{5}_{\mathrm{a}, \mathrm{b}}$ showed potent dose-dependent anticancer efficacy in syngenic murine model (C57Black mice) of melanoma, suppressing cancer growth by an average of $90 \%$ at a dose of $5 \mathrm{mg} / \mathrm{kg}$ by one intra-peritoneum administration at alternate days for 15 days. It also displayed high anticancer activity in the $\mathrm{B} 16$ cells in vitro with nanomolar $\mathrm{IC}_{50}$ value. In addition to the proapoptotic and telomerase inhibition activity previously observed, the compound $\mathbf{5}_{\mathbf{a}, \mathbf{b}}$ has also shown to inhibit cell migration and the deterioration of the actin cytoskeleton. Moreover our spiroketal strongly reduces the HIF1 $\alpha$ expression that is considered as regulator of multiple cellular functions related to the progression from primary to metastatic disease. Therefore, although the full mechanism of action has yet to be completely elucidated, we can conclude that spiroketal $\mathbf{5}_{\mathbf{a}, \mathrm{b}}$ is a promising anticancer drug candidate for the clinical treatment of melanoma.

- Acknowledgements: This study was supported by Regione Autonoma della Sardegna - Italy (research grant Dr. Paola Carta). We thank Dr. Maria Cristina Porcu and Mr. Salvatore Baiano for technical assistance and Mr. Antonio Guicciardi for animal experiments.

- References: 1) Fuggetta, M. P.; De Mico, A.; Cottarelli, A.; Morelli, F.; Zonfrillo, M.; Ulgheri, F.; Peluso, P.; Mannu, A.; Deligia, F.; Marchetti, M.; Roviello, G.; Reyes Romero, A.; Dömling, A.; Spanu, P. Synthesis and Enantiomeric Separation of a Novel Spiroketal Derivative: A Potent Human Telomerase Inhibitor with High in Vitro Anticancer Activity. J. Med. Chem. 2016, 59, 9140-9149.

Tumor Growth Inhibition in Spiroketal Treated Mice

Average Tumor Growth
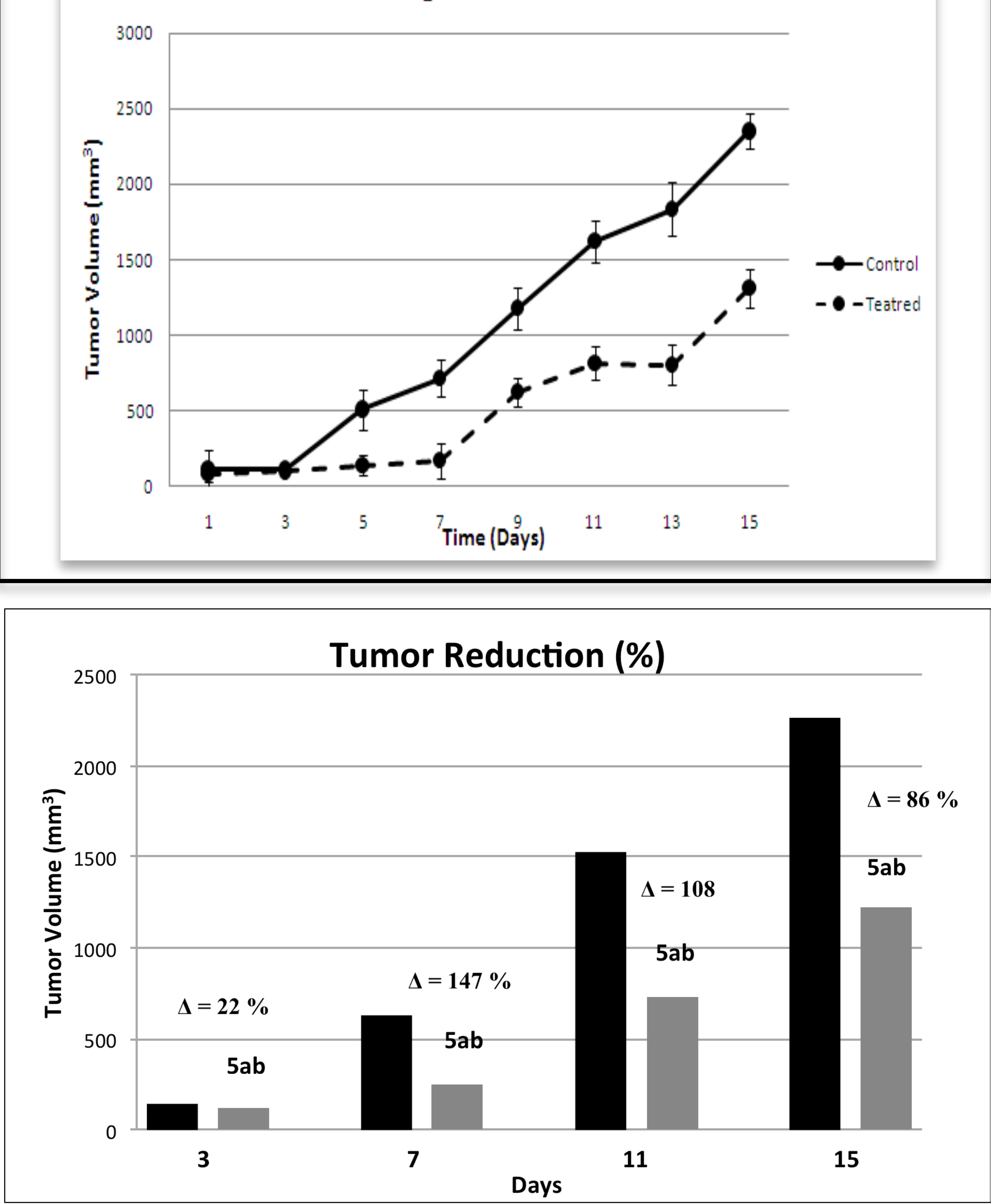